\title{
Molecular trapping in two-dimensional chiral organic Kagomé nanoarchitectures composed of Baravelle spiral triangle enantiomers
}

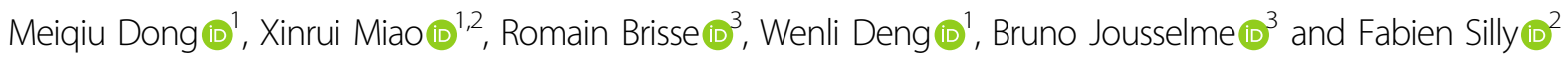

\begin{abstract}
The supramolecular self-assembly of a push-pull dye is investigated using scanning tunneling microscopy (STM) at the liquid-solid interface. The molecule has an indandione head, a bithiophene backbone and a triphenylamine-bithiophene moiety functionalized with two carboxylic acid groups as a tail. The STM images show that the molecules adopt an "L" shape on the surface and form chiral Baravelle spiral triangular trimers at low solution concentrations. The assembly of these triangular chiral trimers on the graphite surface results in the formation of two types of chiral Kagomé nanoarchitectures. The Kagomé-a structure is composed of only one trimer enantiomer, whereas the Kagomé- $\beta$ structure results from the arrangement of two trimer enantiomers in a 1:1 ratio. These Kagomé lattices are stabilized by intermolecular $\mathrm{O}-\mathrm{H} \cdots \mathrm{O} \mathrm{O}$ hydrogen bonds between carboxylic acid groups. These observations reveal that the complex structure of the push-pull dye molecule leads to the formation of sophisticated two-dimensional chiral Kagomé nanoarchitectures. The subsequent deposition of coronene molecules leads to the disappearance of the Kagomé- $\beta$ structure, whereas the Kagomé-a structure acts as the host template to trap the coronene molecules.
\end{abstract}

\section{Introduction}

Engineering novel organic/inorganic interfaces through the self-assembly of functionalized molecules ${ }^{1-18}$ is the focus of intense research interest for developing new applications in nanotechnology ${ }^{19-21}$. "Push-pull" dyes are promising building blocks to fabricate photoactive devices. These dyes are donor-acceptor single compounds; i.e., they possess one electron-rich section as well as one electron-poor section, giving them an intense and tunable absorption band in the visible spectrum. For example, this specific molecular structure has been successfully exploited to enhance the properties of organic solar cells and precious-metal-free dye-sensitized solar cells ${ }^{22,23}$. It has also recently been exploited to develop artificial

Correspondence: Xinrui Miao (msxrmiao@scut.edu.cn) or Fabien Silly (fabien.silly@cea.fr)

${ }^{1}$ School of Materials Science and Engineering, South China University of Technology, Guangzhou 510640, People's Republic of China

2Université Paris-Saclay, CEA, CNRS, SPEC, TITANS, F-91191 Gif sur Yvette, France

Full list of author information is available at the end of the article. photosynthesis devices for low-cost $\mathrm{CO}_{2}$-free hydrogen photo production ${ }^{24}$. Recent progress in the development of more efficient dye-sensitized solar cells and dyesensitized photocathodes is reported in review articles $^{25,26}$. The electronic properties of an organic layer can be drastically modified by the local arrangement of the molecules. It is therefore essential to control molecular assembly to tailor the electronic properties. Hydrogen bonding is an attractive noncovalent interaction that can be used to drive molecular packing. The strength, high selectivity, and directionality of this type of bonding have been successfully exploited to engineer specific organic nanoarchitectures ${ }^{27-34}$. For example, double hydrogen bonds $(\mathrm{O}-\mathrm{H} \cdots \mathrm{O})$ are expected to appear between neighboring molecules possessing carboxylic groups. This strategy has been successfully used to stabilize the formation of porous and compact nanoarchitectures in a highly predictable fashion ${ }^{35-41}$. However, "push-pull" dyes usually have a complex structure with low symmetry. They can also often adopt various conformations. This

\section{(c) The Author(s) 2020}

(c) (i) Open Access This article is licensed under a Creative Commons Attribution 4.0 International License, which permits use, sharing, adaptation, distribution and reproduction cc) in any medium or format, as long as you give appropriate credit to the original author(s) and the source, provide a link to the Creative Commons license, and indicate if changes were made. The images or other third party material in this article are included in the article's Creative Commons license, unless indicated otherwise in a credit line to the material. If material is not included in the article's Creative Commons license and your intended use is not permitted by statutory regulation or exceeds the permitted use, you will need to obtain permission directly from the copyright holder. To view a copy of this license, visit http://creativecommons.org/licenses/by/4.0/. 
means that adding specific molecular substituents to drive molecular assembly does not necessarily imply the formation of ordered and crystalline nanoarchitectures through molecular self-assembly. It is therefore essential to assess molecular arrangements at the atomic scale to elucidate the influence of molecular substituents and molecular conformation on the molecular arrangement. Numerous organic structures can be engineered using molecular self-assembly. The most sophisticated twodimensional (2D) nanoarchitectures are composed of several building blocks, which are themselves composed of several molecules. Archimedean tilings are thus complex structures combining different polygons placed edgeto-edge around a vertex. Stepanenko et al. recently engineered rhombitrihexagonal Archimedean tiling patterns composed of triangles, rhombuses, and hexagons through molecular self-assembly ${ }^{3}$. Trihexagonal tilings, called the Kagomé structure, composed of two hexagons and two triangles sequentially connected to the vertex have also been created $^{42,43}$. To date, noncovalent self-assembled 2D organic Archimedean tilings have been engineered using highly symmetrical molecules with alkyl chains at the liquid-solid interface ${ }^{3,44}$. Engineering sophisticated porous 2D Kagomé and Archimedean tiling nanoarchitectures at the liquid-solid interface using complex unsymmetrical conjugated molecules without alkyl chains remains a challenge. It is also unclear whether these structures can be used as host structures to immobilize guest molecules in a long-range ordered arrangement. The guest molecule should be accepted by the host structure and should not induce a drastic structural transformation of the primary organic template ${ }^{45,46}$.

In this paper, we investigated the self-assembly of a push-pull dye at the 1-octanoic acid/graphite interface. Scanning tunneling microscopy (STM) shows that the molecules adopt an arc conformation and self-assemble into chiral triangular trimers adopting a Baravelle spiral triangle arrangement. These trimers are the building blocks of two 2D chiral Kagomé nanoarchitectures composed of one or two chiral trimers. The addition of coronene molecules leads to the disappearance of one of the Kagomé nanoarchitectures, whereas the second nanoarchitecture acts as a host template.

\section{Methods}

The molecule was synthesized according to the procedure described in ref. ${ }^{47}$. Solutions of the push-pull dye molecule (Fig. 1) in 1-octanoic acid were prepared (concentration: $1 \times 10^{-5}$ to $1 \times 10^{-6} \mathrm{~mol} \mathrm{~L}^{-1}$ ). Droplet of these solutions were then deposited on a highly oriented pyrolytic graphite substrate (HOPG). STM imaging of the samples was performed at the liquid/solid interface using a Nanoscope IIIa Multimode SPM (Bruker, USA) scanning tunneling microscope. Cut $\mathrm{Pt} / \mathrm{Ir}$ tips were used to
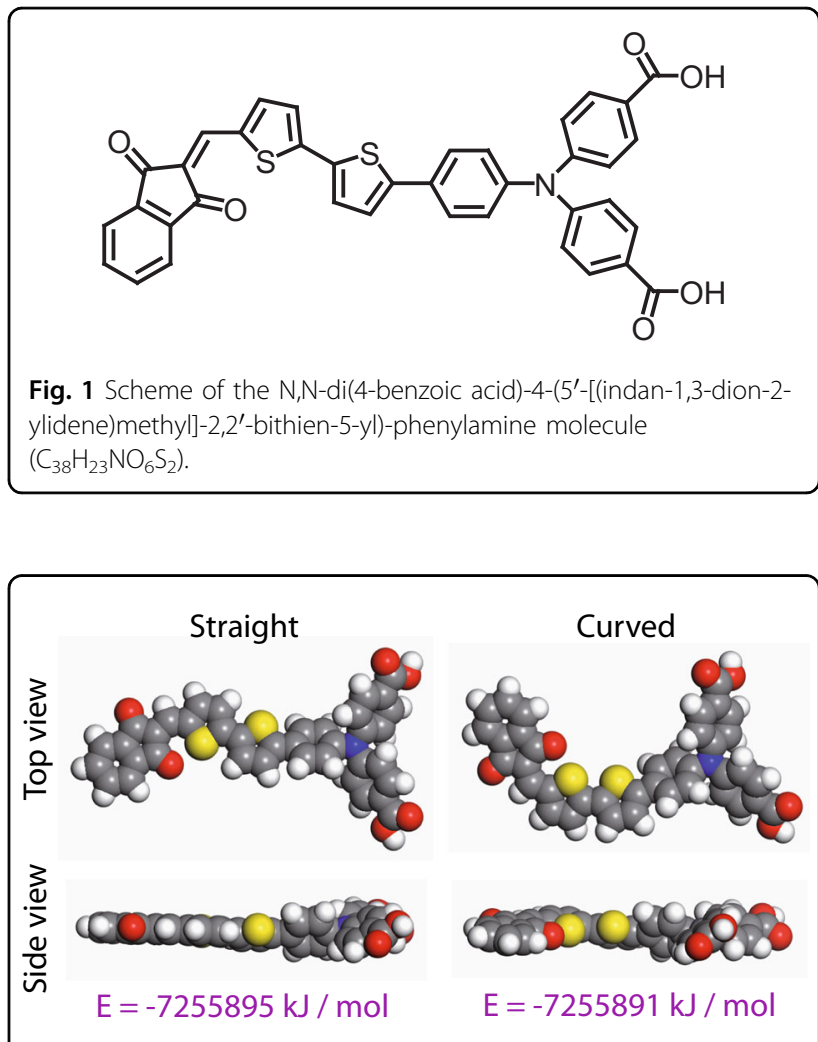

Fig. 2 Scheme of the molecular conformation and corresponding energy. Left: straight conformation. Right: curved conformation. The top and side views of the two molecular conformations are presented.

obtain constant-current images at room temperature with a bias voltage applied to the sample. STM images were processed and analyzed using the application FabViewer ${ }^{48}$.

A scheme of the $N, N$-di(4-benzoic acid)-4-(5'-[(indan1,3-dion-2-ylidene)methyl]-2,2'-bithien-5-yl)-phenylamine molecule is presented in Fig. 1. This molecule is composed of an indandione head, a bithiophene backbone, and a triphenylamine tail. Two carboxylic groups have been added to the triphenylamine group to promote intermolecular hydrogen bonding.

The molecule can adopt different conformations. Density functional theory (DFT) was used to assess the energy of two molecular conformations induced by rotation around $\mathrm{C}-\mathrm{C}$ bond between the thiophene groups. DFT calculations were performed using the Gaussian 09 software package. The full-geometry optimization of the molecule was conducted using the hybrid M06-2X method together with the split-valence polarized $6-31+\mathrm{g}(\mathrm{d})$ basis set. The results are presented in Fig. 2. When the molecule adopts a curved configuration, sulfur atoms of the thiophene groups are adjacent. This curved conformation has a higher conformation energy than the straight conformation, in which one of the thiophene groups is 


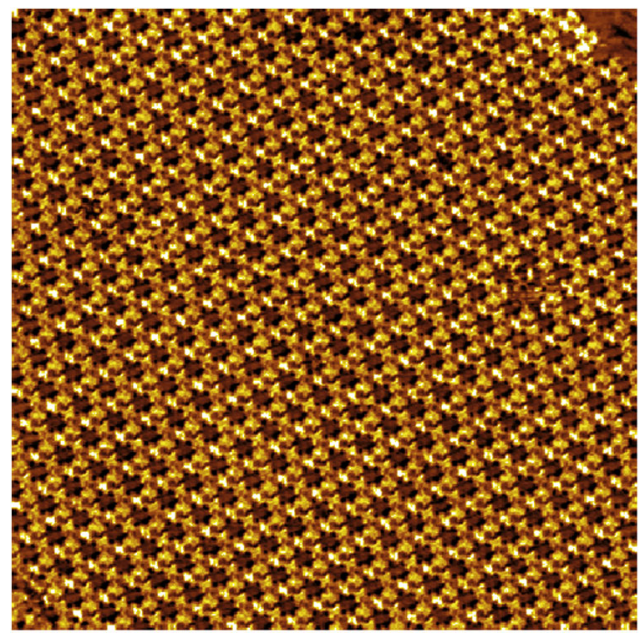

Fig. 3 Self-assembled Kagomé-a nanoarchitecture on graphite. Large-scale STM image, $80 \times 80 \mathrm{~nm}^{2}$ scan area; $\mathrm{V}_{\mathrm{s}}=0.6 \mathrm{~V}, \mathrm{I}_{\mathrm{t}}=400 \mathrm{pA}$.

rotated by $\sim 180^{\circ}$. The energy difference between the two conformations is $\Delta=4 \mathrm{~kJ} \mathrm{~mol}^{-1}$.

\section{Results}

\section{Kagomé-a nanoarchitecture}

The STM image in Fig. 3 shows the molecular layer adsorbed on the graphite surface after deposition of a droplet of the molecule in 1-octanoic acid $\left(1 \times 10^{-6} \mathrm{~mol} \mathrm{~L}^{-1}\right)$. These molecules self-assemble into a large-scale $2 \mathrm{D}$ organic porous hexagonal nanoarchitecture.

A high-resolution STM image of the molecular selfassembly is presented in Fig. 4a. This image reveals that the molecules form a Kagomé- $\alpha$ arrangement. A Kagomé star (white lines) is superimposed onto the porous organic network as a visual guide in Fig. 4a. A high-resolution STM image of a network cavity is presented in Fig. 4b. The STM image shows that the cavities of the Kagomé- $\alpha$ nanoarchitecture are composed of molecular trimers adopting a triangular shape (dashed triangles in Fig. 4b). These trimers are composed of blue-, red-, and greencolored molecules in Fig. 4b. These three molecules form a Baravelle spiral triangle. The model of the molecular arrangement observed in Fig. 4b is presented in Fig. 4d. The molecules adopt an "L" conformation in the trimers. The molecular triphenylamine tails face the center of the network cavities, whereas the molecular indandione heads are located in the center of the triangular trimers. Neighboring trimers appear to be bonded through two pairs of double $\mathrm{O}-\mathrm{H}$... $\mathrm{O}$ hydrogen bonds between the carboxylic groups of neighboring molecules (blue-blue and red-green molecules in Fig. 4d). Neighboring
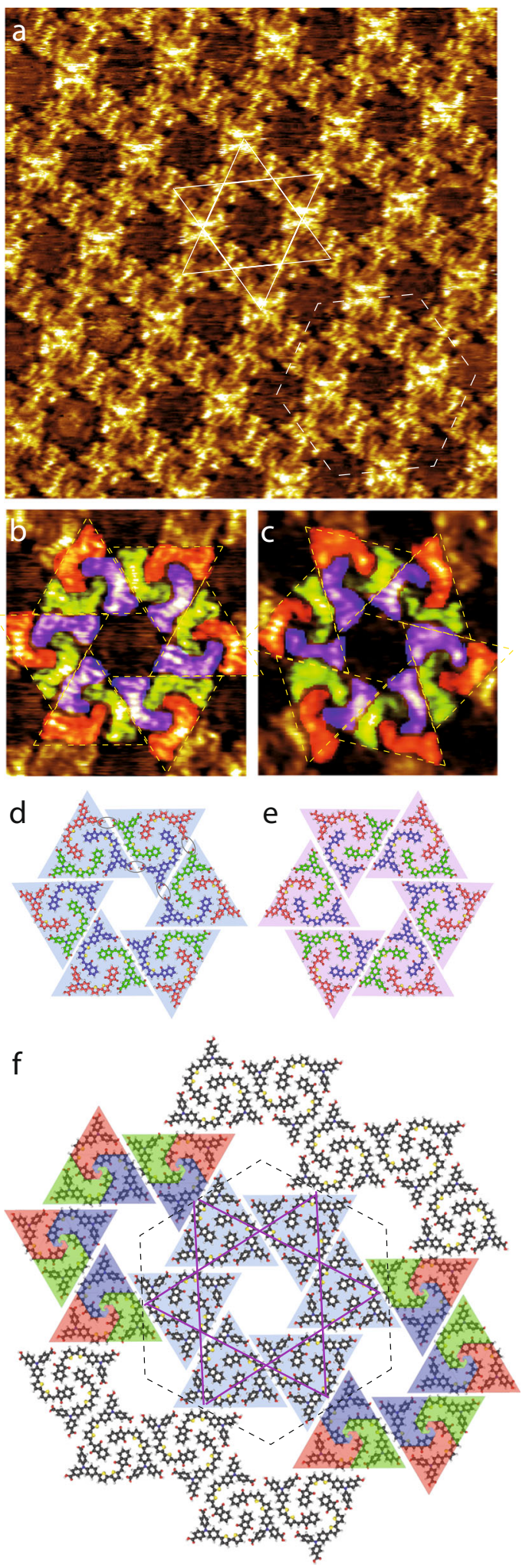


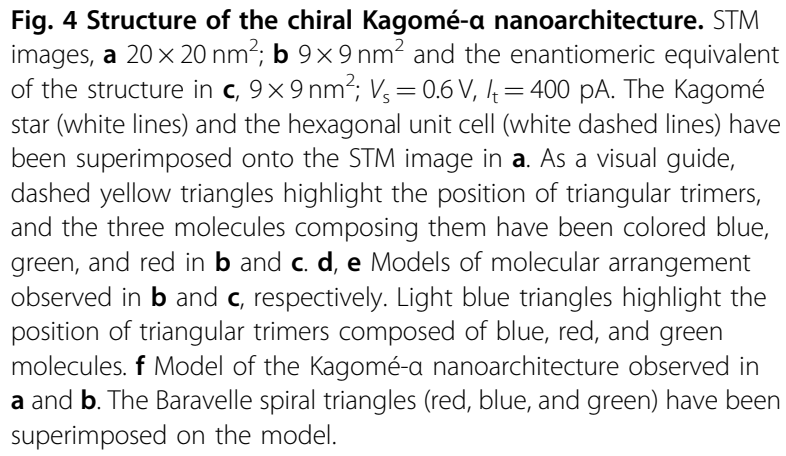

molecules are rotated by $180^{\circ}$ and are arranged side-byside at the trimer boundary (blue and green molecules in Fig. 4d). STM shows that the internal structure of the triangular trimers is chiral and that the arrangement of these trimers is also chiral. The chiral enantiomer of the molecular arrangement observed in Fig. $4 \mathrm{~b}$ is presented in Fig. 4c. The model of the second enantiomer of the Kagomé- $\alpha$ structure is presented in Fig. 4e. The elementary network unit cell of the Kagomé- $\alpha$ structure is a hexagon (represented by dashed black lines in Fig. 4f) with unit cell constants of $4.3 \pm 0.1 \mathrm{~nm}$, and it contains 18 molecules. As a visual guide, superimposed light blue triangles highlight the position of the triangular trimers, whereas the Kagomé- $\alpha$ pattern is represented by pink lines in Fig. 4f. Each enantiomeric Kagomé- $\alpha$ unit cell is only composed of one molecular trimer enantiomer (the distinct trimer enantiomers are highlighted by blue triangles in Fig. 4d and pink triangles in Fig. 4e). The cavities of the Kagomé- $\alpha$ structure have a round shape with a diameter of $2.3 \mathrm{~nm}$.

\section{Kagomé- $\beta$ nanoarchitecture}

STM reveals that a second $2 \mathrm{D}$ porous organic nanoarchitecture is observed at the graphite-solvent interface (Fig. 5). The network unit cell of this structure is now a rectangle (dashed white rectangle superimposed onto the STM image in Fig. 5).

A high-resolution STM image of this second molecular self-assembly is presented in Fig. 6a. This 2D nanoarchitecture is also a Kagomé arrangement (Kagomé- $\beta$ ). A Kagomé star (white lines) is superimposed onto the organic network as a visual guide in Fig. 6a. In contrast to the previous Kagomé- $\alpha$ structure in Fig. 4, the Kagomé- $\beta$ arrangement possesses two cavity shapes. These cavities are highlighted by yellow and pink dashed ellipses in Fig. 6a. High-resolution STM images of these cavities are presented in Fig. 6b (corresponding to the cavity surrounded by a pink dashed ellipse in Fig. 6a) and in Fig. 6c (corresponding to the cavity surrounded by a yellow dashed ellipse in Fig. 6a). The molecules are colored blue,

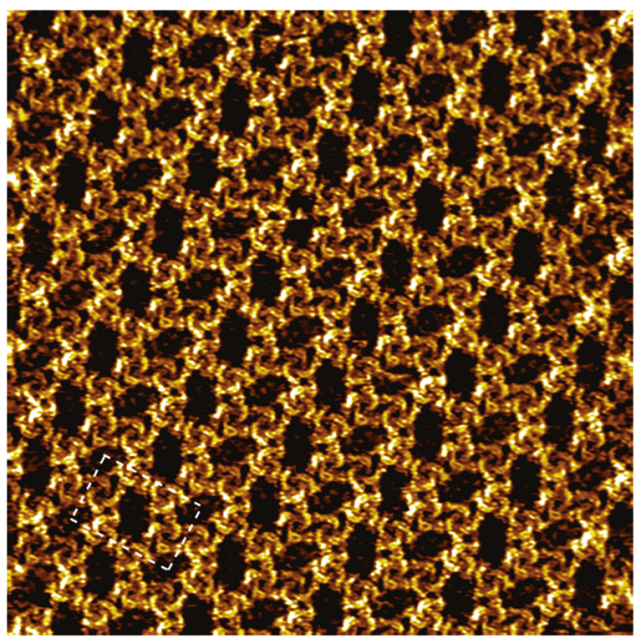

Fig. 5 Self-assembled Kagomé- $\beta$ nanoarchitecture on graphite. Large scale STM image, $40 \times 40 \mathrm{~nm}^{2}$ scan area; $V_{\mathrm{s}}=0.6 \mathrm{~V}, \mathrm{I}_{\mathrm{t}}=400 \mathrm{pA}$.

red, and green as a visual guide. The two cavity shapes are formed by the arrangement of six triangular chiral trimers. The trimer shape is identical to that observed in the Kagomé- $\alpha$ structure in Fig. 4a-c. STM reveals that the Kagomé- $\alpha$ cavities in Fig. 4 b, c are composed of only one trimer enantiomer, whereas the Kagomé- $\beta$ cavities are composed of the two trimer enantiomers (highlighted by blue and pink triangles superimposed onto the STM images in Fig. 6b, c). Two Baravelle spiral triangle enantiomers coexist in the Kagomé- $\beta$ arrangement. Models of the Kagomé- $\beta$ cavities are presented in Fig. $6 \mathrm{~d}$, e. Blue and pink triangles have been placed underneath the different trimer enantiomers as a visual guide. The ratio of the two enantiomers observed in the cavity is $4: 2$ (four pink and two blue triangles in Fig. 6b, $d$ and four blue and two pink triangles in Fig. 6c, e). The side-by-side arrangements of the trimers in the two Kagomé nanoarchitectures are also different. In the cavity of the Kagomé- $\alpha$ arrangement (Fig. 4b, d), molecular triphenylamine tails systematically face the empty center of the cavities. In contrast, only four molecules (blue molecules in the pink triangles) adopt this position in the cavity of the Kagomé- $\beta$ arrangement (Fig. 6b, d) and two molecules (blue molecules in the pink triangles) in the neighboring cavity, as presented in Fig. $6 c$, e. The triphenylamine tail of the other blue molecules faces the bithiophene backbone of the neighboring molecules. The elementary network unit cell of the Kagomé- $\beta$ nanoarchitecture is a rectangle (represented by dashed gray lines in Fig. $6 \mathrm{f}$ ) with $7.6 \pm 0.1 \mathrm{~nm}$ and $4.9 \pm$ $0.1 \mathrm{~nm}$ unit cell constants and contains 12 molecules. As a visual guide, superimposed light blue and pink triangles highlight the position of triangular trimer enantiomers, and the Kagomé star is represented by pink lines in Fig. $6 f$. 


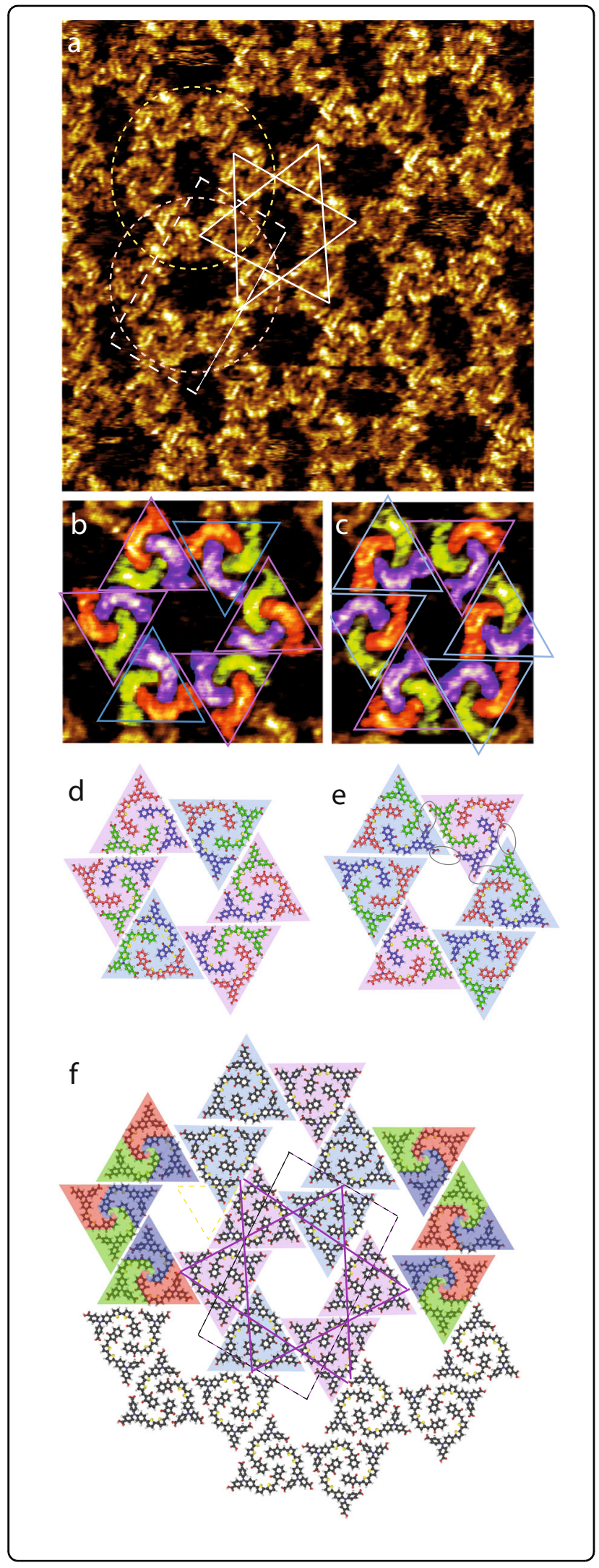

Fig. 6 Structure of the chiral Kagomé- $\beta$ nanoarchitecture. STM images, a $20 \times 20 \mathrm{~nm}^{2} ; \mathbf{b} 8 \times 8 \mathrm{~nm}^{2} ; \mathbf{c} 8 \times 7 \mathrm{~nm}^{2} ; V_{\mathrm{s}}=0.6 \mathrm{~V}, \mathrm{I}_{\mathrm{t}}=400 \mathrm{pA}$. As a visual guide, pink and blue triangles highlight the position of triangular trimer enantiomers, and the three molecules composing them have been colored in blue, green, and red colors in $\mathbf{b}$ and $\mathbf{c} . \mathbf{d}$, e Models of molecular arrangement observed in $\mathbf{b}$ and $\mathbf{c}$, respectively. Light blue triangles highlight the position of triangular trimers

composed of blue, red, and green molecules. $\mathbf{f}$ Model of the Kagomé$\beta$ nanoarchitecture observed in $\mathbf{a}-\mathbf{c}$. The Baravelle spiral triangles (red, blue, and green) have been superimposed on the model.

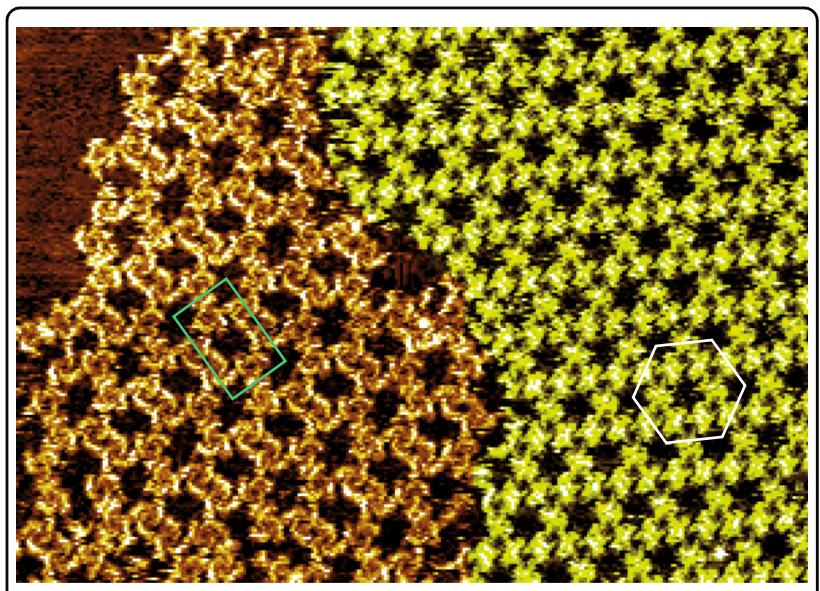

Fig. 7 Coexistence of the two Kagomé nanoarchitectures on graphite. Large-scale STM image, $60 \times 40 \mathrm{~nm}^{2} ; \mathrm{V}_{\mathrm{s}}=0.6 \mathrm{~V}, \mathrm{l}_{\mathrm{t}}=400 \mathrm{pA}$. The Kagomé-a structure is colored in green as a visual guide. The green rectangular and white hexagonal unit cells of the two Kagomé nanoarchitectures have been superimposed on the STM image.

The Kagomé- $\beta$ unit cell is composed of four trimers of the two triangular enantiomers (blue and pink triangles in Fig. 6f). The trimer enantiomer ratio is 1:1. The cavities of the Kagomé- $\beta$ structure have capsule shapes with $1.9 \mathrm{~nm}$ and $3.0 \mathrm{~nm}$ axes.

The large-scale STM image presented in Fig. 7 shows that the two porous Kagomé- $\alpha$ and Kagomé- $\beta$ nanoarchitectures described in Figs. 4 and 6 coexist on the surface.

Molecular concentration can strongly influence molecular self-assembly ${ }^{3,49}$, and molecules without alkyl chains can adopt different packing arrangements ${ }^{50,51}$. The largescale STM image presented in Fig. 8 shows the molecular arrangement on the graphite surface after deposition of a saturated solution of molecules $\left(1 \times 10^{-5} \mathrm{~mol} \mathrm{~L}^{-1}\right)$. The molecules appear to be randomly distributed and randomly arranged on the surface to form a compact layer.

\section{Guest-host nanoarchitecture}

A droplet of solution containing coronene molecules is then deposited on the surface. A nanoarchitecture 

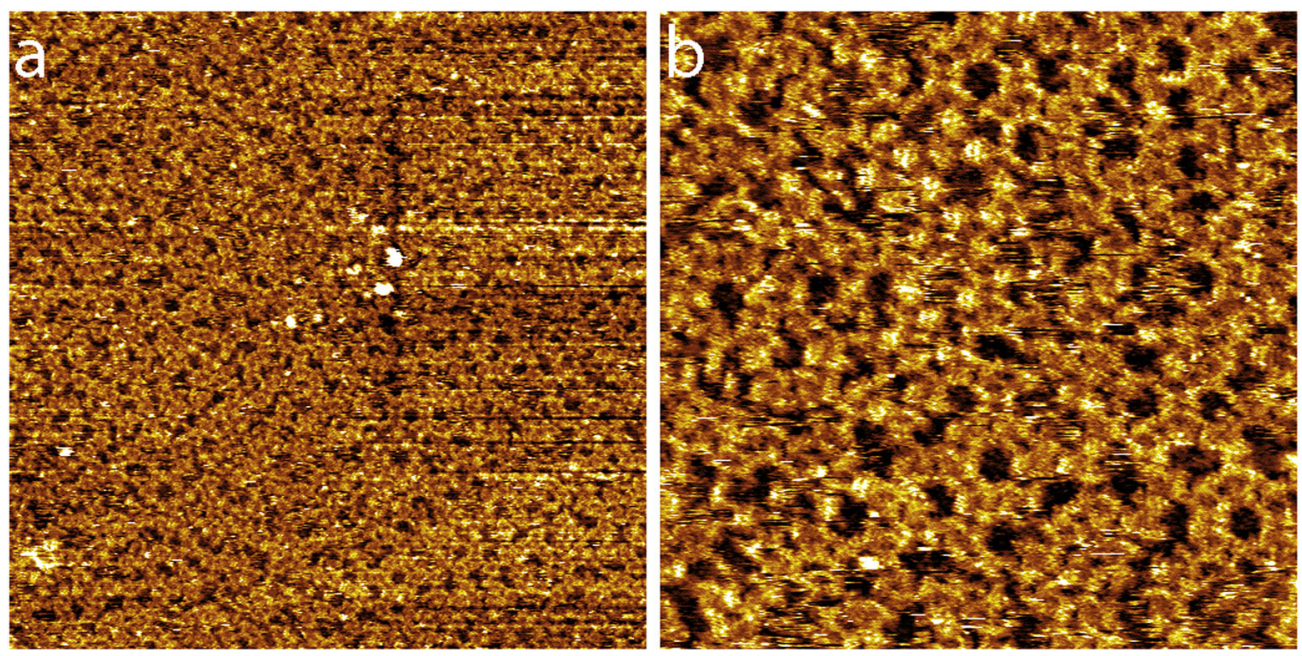

Fig. 8 Molecular assembly at high concentrations. Large-scale STM images, a $100 \times 100 \mathrm{~nm}^{2} ; \mathbf{b} 40 \times 40 \mathrm{~nm}^{2} ; V_{\mathrm{s}}=0.6 \mathrm{~V}, I_{\mathrm{t}}=400 \mathrm{pA}$
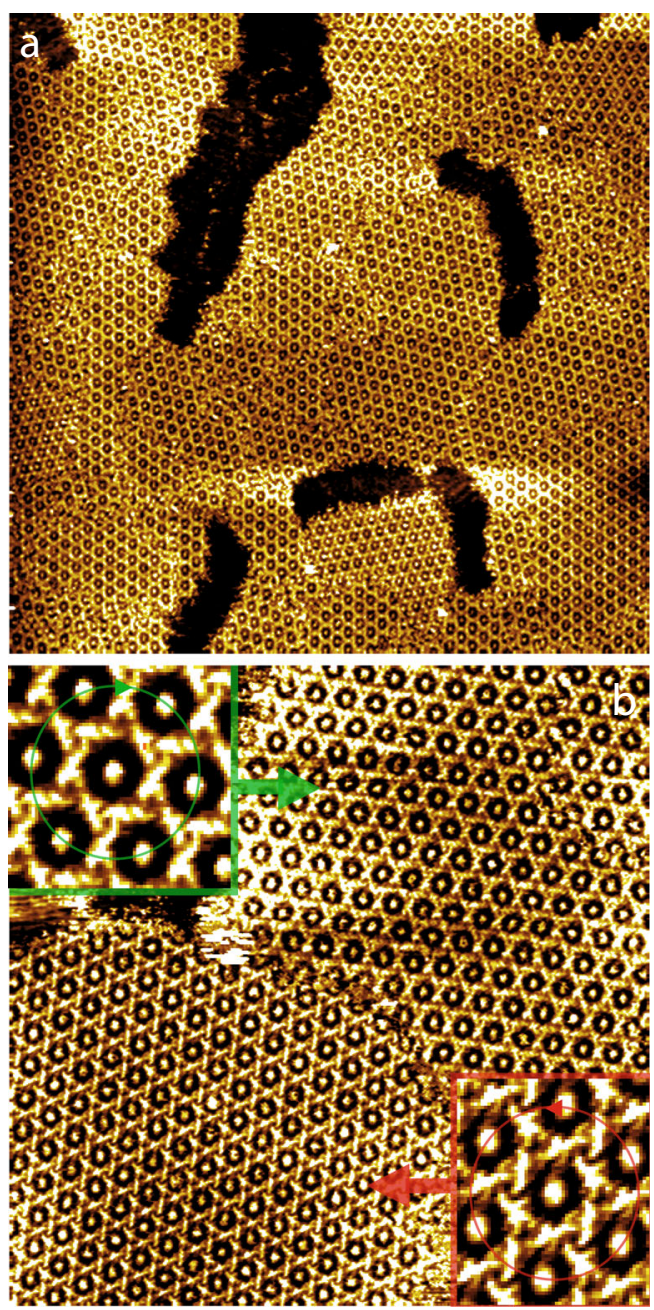

Fig. 9 Molecular coadsorption on graphite. Large-scale STM images, a $200 \times 200 \mathrm{~nm}^{2} ; \mathbf{b} 87 \times 87 \mathrm{~nm}^{2} ; V_{\mathrm{s}}=0.6 \mathrm{~V}, l_{\mathrm{t}}=400 \mathrm{pA}$. featuring bright round spots hexagonally arranged in an organic network is now observed in the large-scale STM image in Fig. 9a. This organic layer is composed of different domains. An STM image of a molecular domain boundary is presented in Fig. 9b. This domain boundary separates two chiral enantiomeric domains (domains of opposite chiral orientation). The chiral enantiomer of each domain is presented in the inset of Fig. 9b-top, left and Fig. 9b-bottom, right. In contrast to the results shown in Fig. 7, only one chiral nanoarchitecture is now observed on the surface.

The high-resolution STM image shown in Fig. 10 reveals that the host structure trapping the coronene molecule is the Kagomé- $\alpha$ nanoarchitecture (the host structure has the same structure and dimensions as the Kagomé- $\alpha$ nanoarchitecture). Only a single guest coronene molecule is hosted inside each cavity of the Kagomé- $\alpha$ network.

\section{Discussion}

STM reveals that the $N, N$-di(4-benzoic acid)-4-(5'[(indan-1,3-dion-2-ylidene)methyl]-2,2'-bithien-5-yl)phenylamine molecule self-assembles into two chiral Kagomé networks on graphite at the solid-liquid interface at low concentrations $\left(\sim 1 \times 10^{-6} \mathrm{~mol} \mathrm{~L}^{-1}\right)$, whereas these molecules do not organize at higher concentrations $(\sim 1 \times$ $10^{-5} \mathrm{~mol} \mathrm{~L}^{-1}$ ). Two Kagomé nanoarchitectures coexist on the surface (Fig. 7). These structures share the same building block. This building block has a triangular shape, and it is composed of three molecules adopting a Baravelle spiral triangular arrangement. Each molecule adopts an "L" conformation in the triangular trimer. It has been previously observed that molecular thiophene chains can adopt a straight conformation ${ }^{52-56}$ or a twisted conformation ${ }^{57}$. The molecular trimer is chiral. The two 

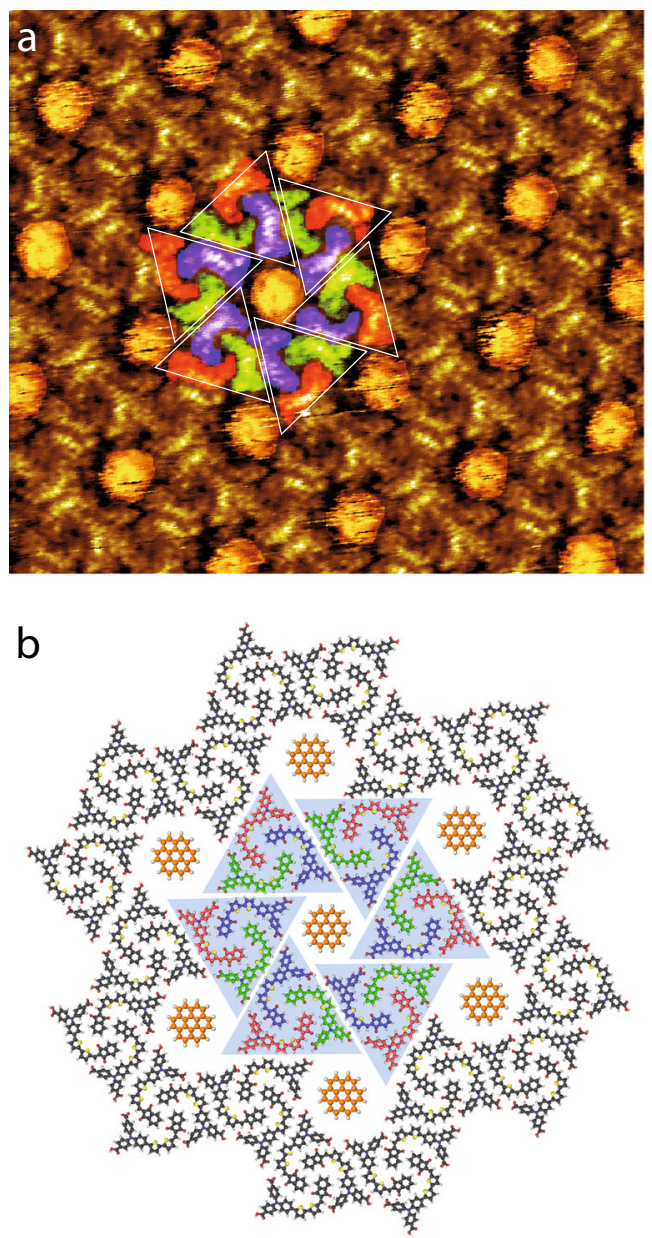

Fig. 10 Structure of the guest-host nanoarchitecture. a Highresolution STM image of the molecular coadsorption on graphite, $20 \times 20 \mathrm{~nm}^{2} ; V_{\mathrm{s}}=0.6 \mathrm{~V}, \mathrm{I}_{\mathrm{t}}=400 \mathrm{pA}$. As a visual guide, molecules have been colored blue, red, and green, whereas coronene molecules have been colored yellow. $\mathbf{b}$ Model of the network cavity, with a coronene molecule in its center.

enantiomers of the trimer have been experimentally observed, as shown in Figs. 4b, c and 6b, c. The Kagomé- $\alpha$ nanoarchitecture is composed of only one trimer enantiomer, as shown in Fig. 4a, f. The second enantiomer is thus only observed in a $2 \mathrm{D}$ arrangement having a mirror symmetric unit cell (compare Fig. 4b with Fig. 4c). In contrast, the two enantiomers coexist in the Kagomé- $\beta$ nanoarchitecture (Fig. $6 \mathrm{~b}-\mathrm{f}$ ). The enantiomer ratio is 1:1; i.e., the trimer enantiomers are equally distributed in the structure. The successful formation of such chiral Kagomé nanoarchitectures depends on fulfilling several molecular conformations and intermolecular interactions: the flexibility and steric arrangement of the backbone, the correct length of the molecules and the carboxylic groups governing the intermolecular arrangement. Interestingly, our results show that all the molecular carboxylic groups form double hydrogen bonds in the Kagomé- $\alpha$ structure, but this is not the case for the Kagomé- $\beta$ structure (see electronic supplementary information, ESI). This suggests that the energy of the Kagomé- $\beta$ structure should be higher than that of the Kagomé- $\alpha$ structure. The fact that the density of the Kagomé- $\alpha$ network $\left(2.7 \mathrm{~nm}^{2}\right.$ per molecule) is slightly lower than that of the Kagomé- $\beta$ network ( $3.1 \mathrm{~nm}^{2}$ per molecule) also suggests that the Kagomé- $\alpha$ network has the lowest-energy structure.

The subsequent deposition of coronene molecules on the surface has a drastic effect on the existence of the two porous Kagomé arrangements. Cavity size and shape can drastically influence the number of species trapped in the porous host structure ${ }^{58}$. The Kagomé- $\beta$ structure disappears, and only the Kagomé- $\alpha$ structure remains on the surface when coronene molecules are also present in the solution. The STM images reveal that the Kagomé- $\alpha$ nanoarchitecture acts as a host structure to trap coronene molecules. All the cavities of the two enantiomeric Kagomé- $\alpha$ nanoarchitectures are filled with guest coronene molecules. This reveals the high efficiency of the two Kagomé- $\alpha$ arrangements to capture coronene molecules (Fig. 9). Figures 5 and 7 show that the cavities of the Kagomé- $\beta$ arrangement are not hexagonal, in contrast to the cavities of the Kagomé- $\alpha$ arrangement. The Kagomé- $\beta$ cavities adopt instead an elliptic shape. It thus appears that the cavity shape of Kagomé- $\beta$ is not adapted to trap coronene molecules. The presence of coronene instead leads to the reorganization of the molecules composing the Kagomé- $\beta$ structure. These molecules will only form the Kagomé- $\alpha$ network, whose cavity shape matches the size and geometry of the coronene molecules (Fig. 10).

\section{Conclusion}

In this paper, we investigated the self-assembly of a push-pull dye on a graphite surface. We showed that the molecule adopts a specific conformation to form chiral Baravelle spiral triangular trimers. These triangular trimers are the building blocks of two complex 2D Kagomé nanoarchitectures that are based on one or two trimer enantiomers. The subsequent deposition of coronene molecules leads to the disappearance of one Kagomé structure, whereas the second structure acts as a host network to trap the coronene molecules inside its cavities. The conformational flexibility of this push-pull dye opens up new opportunities for tailoring molecular selfassembly and then engineering novel organic nanoarchitectures for applications in organic electronic and molecular sensing ${ }^{59-61}$.

\section{Acknowledgements}

Financial support from the Natural Science Foundation of Guangdong Province (2018A030313452), the National Natural Science Foundation of China (21573077), and the Fundamental Research Funds for the Central Universities (SCUT) is gratefully acknowledged. X.R.M. acknowledges receipt of the China Scholarship Council (CSC, 201706155092). The research leading to these results 
has received funding from the European Research Council under the European Union's Seventh Framework Programme (FP7/2007-2013)/ERC grant agreement no. 259297. This work is supported by a public grant overseen by the French National Research Agency (ANR) as part of the "Investissements d'Avenir" program (Labex NanoSaclay, reference: ANR-10-LABX-0035).

\section{Author details}

'School of Materials Science and Engineering, South China University of Technology, Guangzhou 510640, People's Republic of China. ${ }^{2}$ Université ParisSaclay, CEA, CNRS, SPEC, TITANS, F-91191 Gif sur Yvette, France. ${ }^{3}$ Université ParisSaclay, CEA, CNRS, NIMBE, LICSEN, F-91191 Gif sur Yvette, France

\section{Conflict of interest}

The authors declare that they have no conflict of interest.

\section{Publisher's note}

Springer Nature remains neutral with regard to jurisdictional claims in published maps and institutional affiliations.

Supplementary information is available for this paper at https://doi.org/ 10.1038/s41427-020-0201-3.

Received: 26 September 2019 Revised: 20 December 2019 Accepted: 25 December 2019.

Published online: 28 February 2020

\section{References}

1. Seibel, J., Zoppi, L. \& Ernst, K.H. 2D conglomerate crystallization of heptahelicene. Chem. Commun. 50, 8751-8753 (2014).

2. Yang, L., Tan, X., Wang, Z. \& Zhang, X. Supramolecular polymers: historical development, preparation, characterization, and functions. Chem. Rev. 115 7196-7239 (2015)

3. Stepanenko, V., Kandanelli, R., Uemura, S., Würthner, F. \& Fernández, G. Concentration-dependent rhombitrihexagonal tiling patterns at the liquid/ solid interface. Chem. Sci. 6, 5853-5858 (2015).

4. Nguyen, V. Q. et al. Unprecedented self-organized monolayer of a Ru(II) complex by diazonium electroreduction. J. Am. Chem. Soc. 138, 9381-9384 (2016).

5. Zhang, $L$. et al. Site- and configuration-selective anchoring of ironphthalocyanine on the step edges of $\mathrm{Au}(111)$ surface. J. Phys. Chem. C 115 10791-10796 (2011).

6. Seljamäe-Green, R. T. et al. Formation of bioinorganic complexes by the corrosive adsorption of (S)-proline on Ni/Au(111). Langmuir 31, 262-271 (2015).

7. Silly, F. et al. Melamine structures on the Au(111) surface. J. Phys. Chem. C 112 11476-11480 (2008)

8. Skomski, D. et al. High-fidelity self-assembly of crystalline and parallel-oriented organic thin films by П-П stacking from a metal surface. Langmuir $\mathbf{3 0}$ 10050-10056 (2014).

9. Maeda, H. Supramolecular chemistry of pyrrole-based П-conjugated. Molecules. B Chem. Soc. Jpn 86, 1359-1399 (2013).

10. Silly, F. Selecting two-dimensional halogen-halogen bonded self-assembled 1,3,5-tris(4-iodophenyl) benzene porous nanoarchitectures at the solid-liquid interface. J. Phys. Chem. C 117, 20244-20249 (2013).

11. Liu, J. et al. Solvent-controlled 2D host-guest (2,7,12-trihexyloxytruxene/coronene) molecular nanostructures at organic liquid/solid interface investigated by scanning tunneling microscopy. Langmuir 26, 8195-8200 (2010).

12. Yue, J.-Y.; Liu, X.-H.; Sun, B.; Wang, D. The on-surface synthesis of imine-based covalent organic frameworks with non-aromatic linkage. Chem. Commun. 51, 14318-14321 (2015).

13. Wu, R. et al. Self-assembled patterns and young's modulus of single-layer naphthalocyanine molecules on Ag(111). J. Phys. Chem. C 119, 8208-8212 (2015).

14. Qing, H. et al. Atomic force microscopy investigation of growth process of organic TCNQ aggregates on $\mathrm{SiO}_{2}$ and mica substrates. Chin. Phys. B $\mathbf{1 9}$ 080517 (2010)

15. Uemura, S., Aono, M., Sakata, K, Komatsu, T. \& Kunitake, M. Thermodynamic control of $2 \mathrm{D}$ bicomponent porous networks of melamine and melem: diverse hydrogen-bonded networks. J. Phys. Chem. C 117, 24815-24821 (2013).

16. Rosei, F. et al. Properties of large organic molecules on metal surfaces. Progr. Surf. Sci. 71, 95-146 (2003).

17. Yagai, S. Supramolecularly engineered functional $\Pi$-assemblies based on complementary hydrogen-bonding interactions. Bull. Chem. Soc. Jpn 88, 28-58 (2015).

18. Yang, Y. \& Wang, C. Hierarchical construction of self-assembled low-dimensional molecular architectures observed by using scanning tunneling microscopy. Chem. Soc. Rev. 38, 2576 (2009).

19. Yan, H. et al. Rationalization of the selectivity in the optimization of processing conditions for high-performance polymer solar cells based on the polymer self-assembly ability. J. Phys. Chem. C 118, 29473-29481 (2014).

20. Chen, L.-M., Hong, Z., Li, G. \& Yang, Y. Recent progress in polymer solar cells: manipulation of polymer: fullerene morphology and the formation of efficient inverted polymer solar cells. Adv. Mater. 21, 1434-1449 (2009).

21. Fitzner, R. et al. Interrelation between crystal packing and small-molecule organic solar cell performance. Adv. Mater. 24, 675-680 (2012).

22. Qin, P. et al. Design of an organic chromophore for p-type dye-sensitized solar cells. J. Am. Chem. Soc. 130, 8570-8571, 00322 (2008).

23. Li, L., et al. Double-layered NiO photocathodes for p-Type DSSCs with record IPCE. Adv. Mater. 22, 1759-1762 (2010).

24. Li, F. et al. Organic dye-sensitized tandem photoelectrochemical cell for light driven total water splitting. J. Am. Chem. Soc. 137, 9153-9159 (2015).

25. Benazzi, E., Mallows, J., Summers, G. H., Black, F. A. \& Gibson, E. A. Developing photocathode materials for p-type dye-sensitized solar cells. J. Mater. Chem. C 7, 10409-10445 (2019).

26. Gibson, E. A. Dye-sensitizedphotocathodes for $\mathrm{H}_{2}$ evolution. Chem. Soc. Rev. 46, 6194-6209 (2017)

27. Yagai, S. et al. Self-organization of hydrogen-bonding naphthalene chromophores into j-type nanorings and h-type nanorods: impact of regioisomerism. Angew. Chem. Int. Ed. 51, 6643-6647 (2012).

28. Barth, J. V. et al. Building supramolecular nanostructures at surfaces by hydrogen bonding. Angew. Chem. Int. Ed. 39, 1230-1234 (2000).

29. Barth, J. V. Molecular architectonic on metal surfaces. Annu. Rev. Phys. Chem 58, 375-407 (2007).

30. Feyter, S. D. \& Schnyver, F. C. D. Two-dimensional supramolecular self-assembly probed by scanning tunneling microscopy. Chem. Soc. Rev. 32, 139-150 (2003).

31. Bonifazi, D., Mohnani, S. \& Llanes-Pallas, A. Supramolecular chemistry at interfaces: molecular recognition on nanopatterned porous surfaces. Chem. Eur. J. 15, 7004-7025 (2009).

32. $\mathrm{Xu}, \mathrm{W}$. et al. Cyanuric acid and melamine on $\mathrm{Au}(111)$ : structure and energetics of hydrogen-bonded networks. Small 3, 854-858 (2007).

33. $\mathrm{Xu}$, L. et al. Chiral transition of the supramolecular assembly by concentration modulation at the liquid/solid interface. J. Phys. Chem. C 119, 17920-17929 (2015).

34. $\mathrm{Xu}$, L. et al. Concentration-dependent structure and structural transition from chirality to nonchirality at the liquid/solid interface by coassembly. Nanoscale 7. 11734-11745 (2015)

35. Zentner, C. A. et al. High surface area and Z in a thermally stable 8fold polycatenated hydrogen-bonded framework. Chem. Commun. 51, 11642-11645 (2015).

36. Zhang, X., Zeng, Q. \& Wang, C. Molecular templates and nano-reactors: twodimensional hydrogen bonded supramolecular networks on solid/liquid interfaces. RSC Adv. 3, 11351-11366 (2013).

37. Zhou, H. \& Wuest, J. D. Crankshafts: using simple, flat $\mathrm{C}_{2 h}$-symmetric molecules to direct the assembly of chiral $2 \mathrm{D}$ nanopatterns. Langmuir $\mathbf{2 9}, \mathbf{7 2 2 9 - 7 2 3 8}$ (2013).

38. Hu, F.-Y. et al. In situ STM investigation of two-dimensional chiral assemblies through Schiff-base condensation at a liquid/solid interface. ACS Appl. Mater. Inter. 5, 1583-1587 (2013).

39. Suh, M. P., Cheon, Y. E. \& Lee, E. Y. Syntheses and functions of porous metallosupramolecular networks. Coord. Chem. Rev. 252, 1007-1026 (2008).

40. Silly, F. Two-dimensional 1,3,5-tris(4-carboxyphenyl)benzene self-assembly at the 1- phenyloctane/graphite interface revisited. J. Phys. Chem. C 116, 10029-10032 (2012).

41. Miao, K. et al. Hydroxyl versus carboxyl substituent: effects of competitive and cooperative multiple hydrogen bonds on concentration-controlled selfassembly. J. Phys. Chem. C 120, 14187-14197 (2016). 
42. Chen, T., Chen, Q., Zhang, X., Wang, D. \& Wan, L.-J. Chiral Kagomé network from thiacalix[4]arene tetrasulfonate at the interface of aqueous solution/Au (111) surface: an in situ electrochemical scanning tunneling microscopy study. J. Am. Chem. Soc. 132, 5598-5599 (2010).

43. Mo, Y.-P., Liu, X.-H. \& Wang, D. Concentration-directed polymorphic surface covalent organic frameworks: rhombus, parallelogram, and Kagomé. ACS Nano 11, 11694-11700 (2017).

44. Furukawa, S. et al. Molecular geometry directed Kagomé and honeycomb networks: toward two-dimensional crystal engineering. J. Am. Chem. Soc. 128, 3502-3503 (2006)

45. Tahara, K. et al. Harnessing by a diacetylene unit: a molecular design for porous two-dimensional network formation at the liquid/solid interface. Chem. Commun. 50, 2831-2833 (2014).

46. Furukawa, S. et al. Structural transformation of a two-dimensional molecular network in response to selective guest inclusion. Angew. Chem., Int. Ed. 46, 2831-2834 (2007).

47. Brisse, R. et al. A red to blue series of push-pull dyes for NiO based p-DSSCs. Sustain. Energy Fuels 2, 648-654 (2018).

48. Silly, F. A robust method for processing scanning probe microscopy images and determining nanoobject position and dimensions. J. Microsc. 236, 211-218 (2009).

49. Dienstmaier, J. F. et al. On the scalability of supramolecular networks - high packing density vs optimized hydrogen bonds in tricarboxylic acid monolayers. Langmuir 26, 10708-10716 (2010).

50. Li, X. et al. Adsorption and self-assembly of the 2,3,5,6-tetra(2-pyridyl)pyrazine nonplanar molecule on a au(111) surface. J. Phys. Chem. C 120, 6039-6049 (2016).

51. Hooper, J. et al. Chiral surface networks of 3-HPLN - a molecular analog of rounded triangle assembly. Surf. Sci. 629, 65-74 (2014).
52. Azumi, R., Götz, G., Debaerdemaeker, T. \& Bäuerle, P. Coincidence of the molecular organization of $\beta$-substituted oligothiophenes in two-dimensional layers and three-dimensional crystals. Chem. Eur. J. 6, 735-744 (2000).

53. Müller, $\mathrm{H}$. et al. Influence of polar substituents on the epitaxy of oligothiophenes on graphite: a systematic STM investigation. Adv. Mater. 8, 733-737 (1996).

54. Abdel-Mottaleb, M. M. S., Götz, G., Kilickiran, P., Bäuerle, P. \& Mena-Osteritz, E. Influence of halogen substituents on the self-assembly of oligothiophenes-a combined STM and theoretical approach. Langmuir 22, 1443-1448 (2006).

55. Stecher, R., Gompf, B., Münter, J. S. R. \& Effenberger, F. Monolayers of functionalized oligothiophenes on graphite-STM investigation of the influence of intermolecular interactions on the epitaxy. Adv. Mater. 11, 927-931 (1999).

56. Cicoira, F., Miwa, J. A., Melucci, M., Barbarella, G. \& Rosei, F. Ordered assembly of a-quinquethiophene on a copper oxide nanotemplate. Small 2, 1366-1371 (2006).

57. Yang, Z.-Y. et al. Scanning tunneling microscopy of the formation, transformation, and property of oligothiophene self-organizations on graphite and gold surfaces. Proc. Natl Acad. Sci. USA 104, 3707-3712 (2007).

58. Lu, J. et al. Template-induced inclusion structures with copper(II) phthalocyanine and coronene as guests in two-dimensional hydrogen-bonded host networks. J. Phys. Chem. B 108, 5161-5165 (2004).

59. Perepichka, I. F.; Perepichka, D. F. Handbook of Thiophene-Based Materials: Applications in Organic Electronics and Photonics, Vol 2 (John Wiley \& Sons, 2009).

60. Mishra, A., Ma, C.-Q. \& Bäuerle, P. Functional oligothiophenes: molecular design for multidimensional nanoarchitectures and their applications. Chem. Rev. 109, 1141-1276 (2009).

61. Ouchi, $\mathrm{H}$. et al. Impact of helical organization on the photovoltaic properties of oligothiophene supramolecular polymers. Chem. Sci. 9, 3638-3643 (2018). 